Published in final edited form as:

Curr Oncol Rep. ; 22(2): 19. doi:10.1007/s11912-020-0877-0.

\title{
Targeting and therapeutic monitoring of H3K27M-mutant glioma
}

\author{
Kyle Wierzbicki ${ }^{1}$, Karthik Ravi ${ }^{1}$, Andrea Franson ${ }^{1}$, Amy Bruzek ${ }^{2}$, Evan Cantor ${ }^{1}$, Micah \\ Harris $^{3}$, Morgan J. Homan ${ }^{4}$, Bernard L. Marini ${ }^{4}$, Abed Kawakibi ${ }^{1}$, Ramya Ravindran ${ }^{1}$, \\ Rodrigo Teodoro', Vivek Yadav ${ }^{1}$, Carl Koschmann ${ }^{1, *}$ \\ ${ }^{1}$ University of Michigan Medical School, Ann Arbor, MI \\ ${ }^{2}$ Department of Neurosurgery, Michigan Medicine, University of Michigan, Ann Arbor, MI \\ ${ }^{3}$ The Ohio State University College of Medicine, Columbus, $\mathrm{OH}$ \\ ${ }^{4}$ University of Michigan College of Pharmacy, Ann Arbor, MI
}

\section{Abstract}

Purpose of Review: H3K27M is a frequent histone mutation within diffuse midline gliomas and is associated with a dismal prognosis, so much so that the 2016 CNS WHO classification system created a specific category of "Diffuse Midline Glioma, H3K27M-mutant". Here we outline the latest pre-clinical data and ongoing current clinical trials that target $\mathrm{H} 3 \mathrm{~K} 27 \mathrm{M}$, as well as explore diagnosis and treatment monitoring by serial liquid biopsy.

Recent Findings: Multiple epigenetic compounds have demonstrated efficacy and on-target effects in pre-clinical models. The imipridone ONC201 and the IDO1 inhibitor indoximod have demonstrated early clinical activity against H3K27M-mutant gliomas. Liquid biopsy of cerebrospinal fluid has shown promise for clinical use in H3K27M-mutant tumors for diagnosis and monitoring treatment response.

Summary: While H3K27M has elicited a widespread platform of pre-clinical therapies with promise, much progress still needs to be made to improve outcomes for diffuse midline glioma patients. We present current treatment and monitoring techniques as well as novel approaches in identifying and targeting H3K27M-mutant gliomas.

\section{Keywords}

H3K27M; diffuse midline glioma; ONC201; panobinostat; liquid biopsy; circulating tumor DNA

\section{Introduction}

Mutant gliomas and diffuse intrinsic pontine gliomas

In 2016, the World Health Organization (WHO) released a new histological diagnosis in the classification of central nervous system (CNS) malignancies: diffuse midline glioma (DMG), H3K27M-mutant [1]. These WHO grade IV tumors are found in the spinal cord,

*Correspondence to: Carl Koschmann, M.D., Assistant Professor, Pediatric Neuro-Oncology, University of Michigan Medical School, 3520D MSRB I, 1150 W Medical Center Dr, Ann Arbor, MI 48109, Office (p) 734-615-2736 (f) 734-763-2543, Clinic (p) 734-936-9814 (f) 734-232-8740, ckoschma@med.umich.edu. 
brainstem, pineal region, and thalamus and exhibit aggressive clinical behavior [1]. Their distinct classification is the result of a missense mutation caused by a lysine-to-methionine substitution at amino acid position 27 in either histone variant $H I S T 1 H 3 B(\mathrm{H} 3.1)$ or $H 3 F 3 A$ (H3.3) [1]. This mutation results in a unique relatively homogenous phenotype with important genetic, epigenetic, and clinical implications that differ from wildtype DMG and other non-midline high-grade gliomas (HGG) [2, 3]. Both H3.3 and H3.1 variants can be detected with either a CLIA-certified DNA sequencing test or an immune-histochemistry (IHC) stain [1-3].

Diffuse intrinsic pontine glioma (DIPG), a subset of DMG, is a clinical diagnosis made by the radiographic presence of a diffuse tumor comprising at least $50 \%$ of the pons, often in tandem with clinical symptoms, such as cranial neuropathies, necrosis of tissue, and ataxia $[4,5]$. DIPGs that are biopsied are now increasingly referred to by their pathological diagnosis, "diffuse midline glioma +/- H3K27M mutation", as H3K27M status has important therapeutic and prognostic implications within this clinical sub-group [5-7]. Similarly, pediatric and adult non-thalamic high-grade gliomas that were previously referred to by their grade only "anaplastic astrocytoma" or "glioblastoma (GBM)", are now increasingly referred to clinically by this new pathologic diagnosis ("diffuse midline glioma +/- H3K27M mutation").

\section{Epidemiology: age, location, and survival}

H3K27M-mutant DMGs have a bleak prognosis with an overall survival rate of approximately 12 months [4, 8]. Mackay et al. conducted a meta-analysis of 1067 pediatric HGGs and DIPGs and found 63\% of DIPGs and 59.7\% of DMGs to have a positive H3K27M status [8]. Further, H3.3K27M-mutant tumors were found to have a significantly shorter survival time with a median survival of 11.0 months in comparison to the wild type (WT) group with a median survival of 13.5 months; H3.1K27M tumors had a significantly longer median survival of 15.0 months, although multivariate analysis incorporating a variety of clinical factors demonstrated that both $\mathrm{H} 3.1$ and H3.3 mutations are independently associated with shorter overall survival [8]. Hoffman et al. focused on H3K27M-mutant tumors specifically within the pons and found a minimal difference in overall median survival between the mutant group $(n=36)$ and WT group $(n=26)-10.4$ months and 10.5 months, respectively [4]. It is generally understood that $\mathrm{H} 3 \mathrm{~K} 27 \mathrm{M}$ is a poor prognostic variable with very few patients with long term survival.

The majority of H3K27M-mutant tumors are located in the pons, spinal cord, or thalamus and are diagnosed primarily in children and young adults [1]. Recent studies have suggested that the location of these tumors can impact prognosis $[2,9,10]$. Karremann et al. found thalamic H3K27M-mutant DMG patients $(n=24)$ to have a longer median survival than those with H3K27M-mutant DMG located in the spinal cord $(n=6)$, with averages of 13.32 months and 4 months, respectively [2]. Larger studies will need to be done to confirm these findings, as well as determine whether the difference is anatomic/micro-environmental or related to co-occurring mutations.

Historically, H3K27M-mutant DMGs have been diagnosed primarily in children, although these tumors do manifest in adult gliomas. Meyronet et al. analyzed 21 adult H3K27M- 
mutant DMGs, with a median age of diagnosis of 32 years, and found the group to have a median survival of 19.6 months while the WT group of 135 adults, with a median age of diagnosis of 64, had a shorter median survival of 17 months [11]. A recent study in Beijing by Jiang et al. compared radiological, clinical, and molecular characteristics of H3K27Mmutant DMGs in a cohort of 57 adults and 59 children [12]. The mean age was 35.1 years for the adult group and 8.9 years for the pediatric group. The adult cohort had tumors located primarily in the thalamus whereas the pediatric cohort's tumors were predominantly in the brainstem. The median overall survival was 16 months for the adult population and 10 months for the pediatric population [12]. These studies suggest a noticeable difference in clinical behavior of H3K27M-mutant DMGs between adult and pediatric patients, possibly attributed to anatomic/regional difference.

\section{Standard of care for H3K27M-mutant diffuse midline glioma}

In H3K27M-mutant tumors, as in other high-grade gliomas, maximal safe resection is performed when possible. However, this is rarely an option in some midline locations, so small partial resection is attempted when feasible. Following surgical resection, adjuvant radiotherapy using a standard dose of approximately $57 \mathrm{~Gy}$ is administered to patients, with the exception of infants $[5,6,13]$. There is no chemotherapy that has been shown to clearly impact the outcome of patients with H3K27M-mutant DMG. While temozolomide is used concurrently with radiation and adjuvantly at some centers, there are no studies that have demonstrated its clear role in this setting, and it is increasingly understood that $\mathrm{O}^{6}$ methylguanine DNA methyltransferase (MGMT) expression is high in H3K27M-mutant tumors, contributing to temozolomide resistance in H3K27M-mutant DMG [13, 14].

Other than the greater utility of temozolomide in some H3K27M-WT tumors, there is currently no significant difference between the standard of care for H3K27M-mutant and WT tumors. However, there has been an increase in experimental therapies and clinical trials focused on developing stratified treatments. The growing desire for a histological diagnosis of H3K27M in DMGs has resulted in more stereotactic biopsies being performed. These biopsies have been shown to have a high diagnostic yield with minimal effect on patient mortality, contrary to previous beliefs $[15,16]$.

\section{Genetic and epigenetic landscape of H3K27M-mutant DMG}

Among the histone post-translational modifications, the $\mathrm{H} 3$ tail lysine 27 residue plays a pivotal role in the overall functional properties of DNA, and acetylation and methylation results in significant downstream impacts. It is perhaps not surprising that the $\mathrm{H} 3 \mathrm{~K} 27 \mathrm{M}$ mutation results in significant dysfunction of normal epigenetic signaling and programming at the H3K27 locus [7, 17]. Heterozygous (gain of function) H3K27M mutations in either the $H 3 F 3 A$ or $H I S T 1 H 3 B$ gene have been shown to suppress EZH2, a catalytic subunit of polycomb repressive complex 2 (PRC2) which trimethylates the histone $\mathrm{H} 3$ at lysine 27. $\mathrm{H} 3 \mathrm{~K} 27 \mathrm{M}$ inhibition of PRC2 causes a global reduction in total chromatin $\mathrm{H} 3$ dimethylation (K27me2) and trimethylation (K27me3) [7, 18]. This global loss of H3K27 methylation results in both increased cell proliferation potential and decreased differentiation ability (Fig $1 \mathrm{~A})$. 
Spatially and temporally, DIPG and H3K27M DIPGs are felt to be relatively homogenous in terms of primary genetic drivers [19-21]. However, a recent study employing single-cell RNA sequencing (scRNA-seq) on thousands of individual cells from six H3K27M primary gliomas has given us new insight into the cellular and transcriptional architecture of H3K27M tumors [22]. H3K27M gliomas contain a majority of oligodendrocyte precursor cells (OPC) which remain in a state of rapid proliferation, fueling tumor growth and sustained by PDGFRA signaling [23]. The analysis of tumor cells and control normal brain cells showed that PRC2 targets were highly expressed by OPC but were low in oligodendrocyte cells (OC), indicating that for differentiation, OPCs require the PRC2 complex to be functional. Most differentially expressed genes were upregulated in H3K27M gliomas, but some were downregulated [22].

\section{Pre-clinical research and therapeutic vulnerabilities of H3K27M-mutant models}

\section{Evaluation of epigenetic therapies in the context of H3K27M-mutant DIPG tumors}

Global modulation of the methylation/acetylation balance have been considered as new therapeutic approaches to revert the cascade effects generated by the H3K27M mutation as precursor events of tumorigenesis. The most prominent of these attempts is the use of panobinostat, a pan-histone deacetylase inhibitor, which displays inhibition of cellular proliferation and induction of apoptosis in multiple myeloma [23-25]. In H3K27M-mutant tumors, the histone tail poly-acetylation caused by panobinostat can partially rescue H3K27M-induced global hypomethylation by counteracting PRC2 inhibition [25-27].

An alternative approach to restoration of $\mathrm{H} 3 \mathrm{~K} 27 \mathrm{me} 3$ is to inhibit the $\mathrm{H} 3 \mathrm{~K} 27$ demethylase KMD6 (Fig 1A). The experimental demethylase inhibitor GSK-J4 was developed to inhibit JMJD3, a member of the KMD6 gene family, and was found to be a potent inhibitor of cellular proliferation and promoter of apoptosis in K27M-expressing tumor cells in vitro and in orthotopic brain tumor models [28]. Importantly, GSK-J4 synergized with panobinostat in H3.3K27M mutant DIPG cells [28].

While PRC2 function and $\mathrm{H} 3 \mathrm{~K} 27 \mathrm{me} 3$ is globally reduced, other models have established the significance of retained PRC2 activity (and H3K27me3) at focal, but critical, sites which can contribute to proliferative potential in H3K27M-mutant cells by repressing neuronal differentiation and function (Fig 1A) [14]. Indeed, the seemingly paradoxical targeting of EZH2 was efficacious in H3K27M-mutant cells in vitro, by displacing CDKN2A-dependent growth [13].

Finally, bromodomain and extra-terminal (BET) protein inhibitors have also been explored in the epigenetic targeting of $\mathrm{H} 3 \mathrm{~K} 27 \mathrm{M}$ cells. BET inhibitors work through significant target reduction of $\mathrm{H} 3 \mathrm{~K} 27$ acetylation (H3K27ac) levels and accompanying upregulation of differentiation markers (Fig 1A). This approach substantially extended survival of mice harboring orthotopic K27M tumors, even surpassing the effects of GSK-J4 in vivo [29]. Of note, this study provided an alternate explanation of PRC2 inhibition by H3K27M, stating 
that the binding of PRC2 to chromatin is excluded by aberrant locus-specific formation and enrichment in H3K27M-K27ac heterotypic nucleosomes [29].

In the scRNA-seq study by Filbin et al, the PRC1 subunit BMI1, a known oncogene, was found to be one of the few consistently upregulated genes in H3K27M-tumor cells. Suppression of BMI1 in patient-derived cells harboring the H3K27M mutation by means of CRISPR knockout or by pharmacological inhibition reduced the viability of these cells as compared to the control cells [13]. It was also shown that when PDGFRA was knocked out, H3K27M-mutant cells from two of the cell lines showed significant reduced viability, and a combination of both BMI1 inhibition and PDGFRA knockout showed a further reduction in cell viability [13].

Altogether, the wide scope of potentially opposing epigenetic agents with efficacy in H3K27M cells reinforces both the promise and the challenge of targeting the intricate, dynamic balance of gene expression in epigenetically-driven cancers, such as H3K27MDMG.

\section{CAR-T based therapy for H3K27M-mutant DIPG}

Recent work has explored an alternate approach to targeting H3K27M-mutant DMG, through the use of chimeric antigen receptor (CAR) T cells (Fig 1C). CAR T-cell therapy involves the ex vivo genetic modification of patient's autologous $\mathrm{T}$ cells to express a chimeric antigen receptor directed towards a tumor-specific antigen, followed by ex vivo CAR T-cell expansion and re-infusion back into the patient. Anti-CD19 CAR T cells have been FDA-approved based on their efficacy in refractory pediatric acute lymphoblastic leukemia (ALL). The efficacy of CAR T-cell therapy beyond ALL depends upon finding an optimal antigen specific to the individual tumor. In adult glioblastoma, CAR T cells engineered against human epidermal growth factor receptor 2 (EGFR2), which is highly expressed on the surface of a subset of glioblastomas, have shown early anecdotal efficacy in adult GBM [30].

To identify possible antigens for CAR T-cell therapy in DIPG tumors, a recent study observed that patient-derived H3K27M-mutant glioma cell cultures and human tumor tissue had very high expression of the disialoganglioside GD2 (a group of galactose-containing complex-lipid structures found on membranes of cells) which was not observed in nontumor cells [31]. In cultured cells, anti-GD2-CAR T cells showed significant antigendependent cytokine production and killing of H3K27M-mutant DIPG cells, which was not seen in a H3K27-WT cell culture. Anti-GD2-CAR T cells were efficacious in orthotopically engrafted H3K27M tumors (brainstem, thalamic, and spinal cord tumors), except a residual GD2-low expressing cells. Importantly, their model demonstrated significant toxicity in CAR T-treated mice, which is a potential concern with the translation of immune-based therapies for human patients with midline brain tumors [31].

\section{Clinical trials: current and future concepts}

An active area of clinical research in patients with HGGs is in the use of histone deacetylase (HDAC) inhibitors. The HDAC inhibitors vorinostat and panobinostat have been clinically 
tested with favorable safety profiles in children, to date. While there have been some concerns as to the penetration of panobinostat across the blood-brain barrier (BBB) [32], our group has recently obtained murine data demonstrating clinically-significant concentrations of panobinostat within brain tissue (unpublished). Based on its efficacy in pre-clinical $\mathrm{H} 3 \mathrm{~K} 27 \mathrm{M}$ models, there is currently a phase 1 trial evaluating the safety of panobinostat administration in children and young adults with DIPG through the Pediatric Brain Tumor Consortium [33]. Other phase 1 trials include a recently-opened trial of fimepinostat, a combined HDAC and phosphoinositide 3 kinase (PI3k) inhibitor in children and young adults with DIPG, HGG, or medulloblastoma [34]. A phase 1 trial of combination therapy with vorinostat and the mTOR inhibitor temsirolimus, with and without radiotherapy, in children and young adults with DIPG is ongoing [35].

ONC201 is an investigational molecule that antagonizes dopamine receptor D2 (DRD2) and was first identified in a screen for compounds that induce the cytokine TNF-related apoptosis-inducing ligand (TRAIL) [36, 37]. ONC201 was reported to inactivate kinases Akt and ERK which leads to Foxo3a-driven upregulation of TRAIL in the nucleus and promotes cancer cell death through activation of the integrated stress response [37, 38]. ONC201 has shown promise in the clinical setting as an anti-tumor agent with high CNS penetration and is currently being investigated in clinical trials for adult and pediatric H3K27M glioma, including one expanded access protocol [39-42].

In a patient-derived cell viability panel, ONC201 potency was highest against H3K27Mmutant glioma with reduced activity against wildtype glioma cell lines [43]. ONC201 clinical efficacy in adult H3K27M-mutant glioma was first demonstrated in a 22-year-old patient who experienced durable $96 \%$ objective response [44, 45]. Furthermore, significant clinical and radiographic response has been reported in a DIPG patient treated with ONC201 [46]. Based on this early promising data, Oncoceutics (the manufacturer of ONC201), is applying for accelerated FDA approval, which if granted, would be the first agent with that status for H3K27M-mutant glioma. Multiple phase 2 trials with ONC201 are being developed for H3K27M-mutant glioma through the Children's Oncology Group (COG) and the Pacific Neuro-Oncology Consortium (PNOC).

Indoleamine-2,3-dioxygenase 1 (IDO1) is an enzyme that catabolizes tryptophan to kynurenine, leading to an immunosuppressive tumor microenvironment [47]. As a result, clinical trial focus has been on the combination of immune checkpoint inhibition with IDO1 inhibition [48]. IDO1 expression was found to be high in tumor samples from pediatric patients with HGG, and pre-clinical work demonstrated potent inhibition of IDO1 with the BBB-penetrating IDO1 inhibitor BGB-5777 [49]. In a murine glioma model, IDO1 inhibition in combination with temozolomide demonstrated synergistic anti-tumor activity, and the combination of the IDO1 inhibitor indoximod and temozolomide combined with radiation therapy is currently being studied in children with newly-diagnosed DIPG, with promising early clinical results $[6,50,51]$.

As mentioned, there has been a significant amount of interest in the development of CAR T cells as a treatment for solid tumors. Previous work on the immune microenvironment of DIPG tumors has been found to be rather neutral, with low chemokine and cytokine 
expression and lack of macrophage or $\mathrm{T}$ cell infiltration, yet they lack highly immunosuppressive signatures as seen in other tumors $[52,53]$. Preclinical work with CAR $\mathrm{T}$ cells targeting B7-H3, an immune checkpoint regulator, or GD2 in HGGs is promising [54-56]. A clinical trial with these GD2-CAR T cells is in development at Stanford University, and the upcoming Brainchild-03 trial (Seattle Children's Hospital) will utilize B7-H3 targeted CAR T cells with infusion of CAR T cells via an intratumoral catheter.

Overcoming the BBB and achieving therapeutic levels of medication in the tumor continues to be a challenge in DIPG treatment. Though limited by the ability to place catheters into tumors and keep proper positioning, convection enhanced delivery (CED) has been explored as a promising method of directly delivering therapeutic levels of medication while limiting systemic side effects (Fig 1B) [57, 58]. Currently open phase 1/2 trials utilizing CED in DIPG include a study of a panobinostat nanoparticle formulation along with gadoteridol in newly diagnosed DIPG patients, as well as a trial delivering nanoliposomal irinotecan following radiotherapy $[59,60]$. A study open since 2011 has utilized CED to deliver a radionucleotide-antibody complex that targets the $\mathrm{B} 7-\mathrm{H} 3$ antigen commonly expressed by DIPG [61]. While promising, many issues remain in wider use of CED in DMG. In order to employ CED, the drug itself has to be soluble, and many promising targeted drugs (e.g. tyrosine kinase inhibitors), don't have an aqueous/IV formulation. As well, the logistics and legal issues involved with scaling such a technically complicated therapy have limited its widespread use. Nevertheless, investigators continue to pursue this option, possibly with future use for "local control" of DMG, alongside systemic therapy, in order to reduce risk of re-growth outside of the CED field.

\section{Therapeutic monitoring and the utility of liquid biopsies}

Selection of appropriate treatment plans and palliative care options for HGGs are heavily dependent on therapeutic monitoring to infer response to therapy and burden of disease. Contrast-enhanced MRI is routinely considered the gold standard approach, providing a non-invasive method to image and track tumor progression. Despite advanced imaging techniques, such as proton magnetic resonance spectroscopy or dynamic susceptibility contrast MRI which allow for important metabolic and tumor tissue perfusion analysis, significant limitations still exist [62]. Radiological changes during or after treatment, such as volume expansion or increase in FLAIR signal or contrast enhancement, may be from a variety of causes, including pseudoprogression, pseudoregression, and radionecrosis [62, 63]. This contributes to a lack of sensitivity and specificity in regard to MR imaging. As therapeutic decisions are guided by changes in tumor growth, it is of paramount importance that an accurate and minimally-invasive method be developed to monitor treatment response. Current research is being done to assess whether liquid biopsy for $\mathrm{H} 3 \mathrm{~K} 27 \mathrm{M}$ is a feasible means by which to do so.

Brain tumor cells release circulating tumor DNA (ctDNA) into surrounding cerebrospinal fluid (CSF) and can cross the BBB into plasma [64]. This cellular mechanism has important clinical utility for diagnostic tests, genetic profiling of tumors, and therapeutic monitoring. ctDNA has already been used to track disease progression in adult cancers including liver, 
lung, and breast cancers; the application of this tool to the pediatric CNS population is presently underway [65-67].

Digital droplet PCR (ddPCR) is an ultrasensitive PCR method allowing for detection and quantification of ctDNA at extremely low concentrations. PCR reactions are partitioned into thousands of droplets, allowing for highly sensitive and rapid quantification of low mutant allele frequencies (MAF) [68]. As H3K27M is the most frequent mutation in pediatric brainstem gliomas and is closely associated with clinical outcomes, it has been the subject of much ddPCR experimentation $[2,69,70]$.

Sequencing of ctDNA in CSF also allows investigators to detect tumor mutations [70]. The advantage that sequencing has over ddPCR is the ability to detect innumerable mutations at once, including but not limited to H3K27M, with high sensitivity and specificity. The use of sequencing to diagnose and monitor treatment response of tumor genetics in CSF is ongoing and holds great promise.

ctDNA can be correlated to standard brain imaging measurements to track, and potentially even predict, changes in tumor growth. Mutant H3K27M copies from CSF of pediatric DIPG patients is positively correlated with contrast-enhancing cross-sectional tumor area on MRI [71]. Further, a decrease of H3K27M MAF by both ddPCR analysis and sequencing was observed following radiotherapy treatment. The ddPCR and sequencing results correlated with a decrease in tumor burden as measured by MRI tumor volume [63]. These novel findings emphasize the therapeutic monitoring value of ctDNA and the utility of the ddPCR platform. The specificity and sensitivity of ddPCR and sequencing of tumor mutations in CSF elicit the possibility of leveraging ctDNA as an early biomarker for disease progression, preceding visible tumor response by MRI $[67,72,73]$. This could allow for earlier and more effective therapeutic decision-making to occur in the clinical setting.

These preliminary data demonstrate the feasibility and promise of liquid biopsies to monitor treatment response in pediatric DMG patients. Current barriers to wider spread use include adoption of serial lumbar puncture as standard of care for surveillance of DMG and the expansion of CLIA-certified labs offering liquid ctDNA analysis [74].

\section{Conclusion}

The histone mutation H3K27M has served as a catalyst within the field of neuro-oncology, providing a vulnerable mechanism of which new therapeutic techniques have taken advantage. Though clinical trials are still in their early phases and have yet to produce definitive data, there is nonetheless an exciting amount of anticipation regarding these recently translated concepts. H3K27M ctDNA has also provided a means by which to accurately and sensitively monitor and quantify disease burden, potentially supplementing or substituting conventional imaging and surgical procedures. DMGs pose a lethal prognosis due to the lack of surgical intervention and ineffectiveness of current drug and radiation therapies. However, with the advent of a further understanding of the biology of H3K27M, the future landscape of precision medicine and outlook for DMG patients appears hopeful. 


\section{References}

1. Louis DN, Perry A, Reifenberger G, von Deimling A, Figarella-Branger D, Cavenee WK, et al. The 2016 World Health Organization classfication of tumors of the central nervous system: a summary. Acta Neuropathol. 2016;131(6):803-820. doi:10.1007/s00401-016-1545-1. [PubMed: 27157931]

2. Karremann M, Gielen GH, Hoffmann M, Wiese M, Colditz N, Warmuth-Metz M, et al. Diffuse high-grade gliomas with $\mathrm{H} 3 \mathrm{~K} 27 \mathrm{M}$ mutations carry a dismal prognosis independent of tumor location. Neuro Oncol. 2018;20(1):123-31. doi:10.1093/neuonc/nox149. [PubMed: 29016894]

3. Sturm D, Witt H, Hovestadt V, Khuong-Quang DA, Jones DT, Konermann C, et al. Hotspot mutations in H3F3A and IDH1 define distinct epigenetic and biological subgroups of glioblastoma. Cancer Cell. 2012;22(4):425-37. doi:10.1016/j.ccr.2012.08.024. [PubMed: 23079654]

4. Hoffman LM, Veldhuijzen van Zanten SEM, Colditz N, Baugh J, Chaney B, Hoffmann M, et al. Clinical, radiologic, pathologic, and molecular characteristics of long-term survivors of diffuse intrinsic pontine glioma (DIPG): a collaborative report from the international and european society for pediatric oncology DIPG registries. J Clin Oncol. 2018;36(19):1963-72. [PubMed: 29746225]

5. Cohen KJ, Jabado N, Grill J. Diffuse intrinsic pontine gliomas-current management and new biologic insights. Is there a glimmer of hope? Neuro Oncol. 2017;19(8):1025-34. doi:10.1093/ neuonc/nox021. [PubMed: 28371920]

6. Hargrave D, Bartels U, Bouffet E. Diffuse brainstem glioma in children: critical review of clinical trials. Lancet Oncol. 2006;7(3):241-8. [PubMed: 16510333]

7. Lulla RR, Saratsis AM, Hashizume R. Mutations in chromatin machinery and pediatric high-grade glioma. Sci Adv. 2016;2(3):e1501354. doi:10.1126/sciadv.1501354. [PubMed: 27034984]

8. Mackay A, Burford A, Carvalho D, Izquierdo E, Fazal-Salom J, Taylor KR, et al. Integrated molecular meta-analysis of 1,000 pediatric high-grade and diffuse intrinsic pontine glioma. Cancer Cell. 2017;32(4):520-37 e5. doi:10.1016/j.ccell.2017.08.017. [PubMed: 28966033] • This largescale study investigated the characteristic biological and clinical differences between H3K27Mmutant and H3K27-WT DMGs, demonstrating that the histone mutation has poor prognistic implications in pediatric populations.

9. Liu Y, Chen H, Shou W. Potential common pathogenic pathways for the left ventricular noncompaction cardiomyopathy (LVNC). Pediatr Cardiol. 2018;39(6):1099-106. doi:10.1007/ s00246-018-1882-z. [PubMed: 29766225]

10. Pratt D, Natarajan SK, Banda A, Giannini C, Vats P, Koschmann C, et al. Circumscribed/nondiffuse histology confers a better prognosis in H3K27M-mutant gliomas. Acta Neuropathol. 2018;135(2):299-301. doi:10.1007/s00401-018-1805-3. [PubMed: 29302777]

11. Meyronet D, Esteban-Mader M, Bonnet C, Joly MO, Uro-Coste E, Amiel-Benouaich A, et al. Characteristics of H3 K27M-mutant gliomas in adults. Neuro Oncol. 2017;19(8):1127-34. doi:10.1093/neuonc/now274. [PubMed: 28201752]

12. Jiang H, Yang K, Ren X, Cui Y, Li M, Lei Y, et al. Diffuse midline glioma with an H3 K27M mutation: a comparison integrating the clinical, radiological, molecular features between adult and pediatric patients. Neuro Oncol. 2019. doi:10.1093/neuonc/noz152.

13. Abe H, Natsumeda M, Kanemura Y, Watanabe J, Tsukamoto Y, Okada M, et al. MGMT expression contributes to temozolomide resistance in H3K27M-mutant diffuse midline gliomas and MGMT silencing to temozolomide sensitivity in IDH-mutant gliomas. Neurol Med Chir (Tokyo). 2018;58(7):290-295. doi:10.2176/nmc.ra.2018-0044. [PubMed: 29848907]

14. Guerra-Garcia P, Marshall LV, Cockle JV, Ramachandran PV, Saran FH, Jones C, et al. Challenging the indiscriminate use of temozolomide in pediatric high-grade gliomas: a review of past, current, and emerging therapies. Pediatr Blood Cancer. 2019;e28011. doi:10.1002/pbc.28011. [PubMed: 31617673]

15. Hamisch C, Kickingereder P, Fischer M, Simon T, Ruge MI. Update on the diagnostic value and safety of stereotactic biopsy for pediatric brainstem tumors: a systematic review and meta-analysis of 735 cases. J Neurosurg Pediatr. 2017;20(3):261-8. doi:10.3171/2017.2.PEDS1665. [PubMed: 28621573] 
16. Pincus DW, Richter EO, Yachnis AT, Bennett J, Bhatti MT, Smith A. Brainstem stereotactic biopsy sampling in children. J Neurosurg. 2006;104(2 Suppl):108-14. doi:10.3171/2017.2.PEDS1665. [PubMed: 16506498]

17. Maury E, Hashizume R. Epigenetic modification in chromatin machinery and its deregulation in pediatric brain tumors: insight into epigenetic therapies. Epigenetics. 2017;12(5):353-69. doi:10.1080/15592294.2016.1278095. [PubMed: 28059591]

18. Piunti A, Hashizume R, Morgan MA, Bartom ET, Horbinski CM, Marshall SA, et al. Therapeutic targeting of polycomb and BET bromodomain proteins in diffuse intrinsic pontine gliomas. Nat Med. 2017;23(4):493. doi:10.1038/nm.4296. [PubMed: 28263307]

19. Koschmann C, Farooqui Z, Kasaian K, Cao X, Zamler D, Stallard S, et al. Multi-focal sequencing of a diffuse intrinsic pontine glioma established PTEN loss as an early event. NPJ Precis Oncol. 2017;1(1):32. doi:10.1038/s41698-017-0033-y. [PubMed: 29872713]

20. Salloum R, McConechy MK, Mikael LG, Fuller C, Drissi R, DeWire M, et al. Characterizing temporal genomic heterogeneity in pediatric high-grade gliomas. Acta Neuropathol Commun. 2017;5(1):78. doi:10.1186/s40478-017-0479-8. [PubMed: 29084603]

21. Hoffman LM, DeWire M, Ryall S, Buczkowicz P, Leach J, Miles L, et al. Spatial genomic heterogeneity in diffuse intrinsic pontine and midline high-grade glioma: implications for diagnostic biopsy and targeted therapeutics. Acta Neuropathol Commun. 2016;4:1. doi:10.1186/ s40478-015-0269-0. [PubMed: 26727948]

22. Filbin MG, Tirosh I, Hovestadt V, et al. Developmental and oncogenic programs in H3K27M gliomas dissected by single-cell RNA-seq. Science. 2018;360(6386):331-335. doi:10.1126/ science.aao4750. doi:10.1126/science.aao4750. [PubMed: 29674595]

23. Grasso CS, Tang Y, Truffaux N, Berlow NE, Liu L, Debily MA, et al. Functionally defined therapeutic targets in diffuse intrinsic pontine glioma. Nat Med. 2015;21(6):555-9. doi:10.1038/ nm0715-827a. [PubMed: 25939062] • This study emphasizes panobinostat as a promising drug capable of partially rescuing the global hypotrimethylation of the H3K27M phenotype in vitro. Further analysis revealed synergic effects when DIPG cell cultures were treated with both panobinostat and GSK-J4.

24. Brown ZZ, Müller MM, Jain SU, Allis CD, Lewis PW, Muir TW. Strategy for “detoxification” of a cancer-derived histone mutant based on mapping its interaction with the methyltransferase PRC2. J Am Chem Soc. 2014;136(39):13498-13501. [PubMed: 25180930]

25. Hashizume R Epigenetic targeted therapy for diffuse intrinsic pontine glioma. Neurologia medicochirurgica. 2017;57(7):331-342. doi:10.2176/nmc.ra.2017-0018. [PubMed: 28592748]

26. Hennika T, Hu G, Olaciregui NG, Barton KL, Ehteda A, Chitranjan A, et al. Pre-clinical study of panobinostat in xenograft and genetically engineered murine diffuse intrinsic pontine glioma models. PLoS One. 2017;12(1):e0169485. doi:10.1371/journal.pone.0169485. [PubMed: 28052119]

27. Kruidenier L, Chung C, Cheng Z, Liddle J, Che K, Joberty G, et al. A selective jumonji H3K27 demethylase inhibitor modulates the proinflammatory macrophage response. Nature. 2012;488(7411):404. doi:10.1038/nature11262. [PubMed: 22842901]

28. Hashizume R, Andor N, Ihara Y, Lerner R, Gan H, Chen X, et al. Pharmacologic inhibition of histone demethylation as a therapy for pediatric brainstem glioma. Nat Med. 2014;20(12):1394. doi:10.1038/nm.3716. [PubMed: 25401693]

29. Mohammad F, Weissmann S, Leblanc B, Pandey DP, Hojfeldt JW, Comet I, et al. EZH2 is a potential therapeutic target for H3K27M-mutant pediatric gliomas. Nat Med. 2017;3(4):483. doi:10.1038/nm.4293.

30. Martinez M, Moon EK. CAR T cells for solid tumors: new strategies for finding, infiltrating, and surviving in the tumor microenvironment. Front Immunol. 2019;10:128. doi:10.3389/ fimmu.2019.00128. [PubMed: 30804938]

31. Mount CW, Majzner RG, Sundaresh S, Arnold EP, Kadapakkam M, Haile S, et al. Potent antitumor efficacy of anti-GD2 CAR T cells in H3-K27M+ diffuse midline gliomas. Nat Med. 2018;24(5):572-9. doi: 10.1038/s41591-018-0006-x. [PubMed: 29662203] • This pre-clinical study demonstrates the efficacy and selectivity of GD2-CAR T cells in both patient-derived DIPG in vitro cell cultures and in vivo mouse models. This novel immunotherapy is promising, but its tolerability in pediatric DMG patients has yet to be demonstrated. 
32. Rasmussen TA, Tolstrup M, Moller HJ, Brinkmann CR, Olesen R, Erikstrup C, et al. Activation of latent human immunodeficiency virus by the histone deacetylase inhibitor panobinostat: a pilot study to assess effects on the central nervous system. Open Forum Infect Dis. 2015;2(1):ofv037. doi:10.1093/ofid/ofv037. [PubMed: 26034779]

33. Trial of Panobionostat in Children With Diffuse Intrinsic Pontine Glioma (PBTC-047).

34. Fimepinostat in Treating Brain Tumors in Children and Young Adults (PNOC016).

35. Vorinostat and Temsirolimus With or Without Radiation Therapy in Treating Younger Patients With Newly Diagnosed or Progressive Diffuse Intrinsic Pontine Glioma.

36. Allen JE, Kline CL, Prabhu VV, Wagner J, Ishizawa J, Madhukar N, et al. Discovery and clinical introduction of first-in-class imipridone ONC201. Onctotarget. 2016;7(45):74380-74392. doi:10.18632/oncotarget.11814.

37. Allen JE, Krigsfeld G, Mayes PA, Patel L, Dicker DT, Patel AS, et al. Dual inactivation of Akt and ERK by TIC10 signals Foxo3a nuclear translocation, TRAIL gene induction, and potent antitumor effects. Sci Transl Med. 2013;5(171):171ra17. doi:10.1126/scitranslmed.3004828.

38. Kline CL, Van den Heuvel AP, Allen JE, Prahbu VV, Dicker DT, El-Deiry WS. ONC201 kills solid tumor cells by triggering an integrated stress response dependent on ATF4 activation by specific elF2a kinases. Sci Signal. 2016;9(415):ra18. [PubMed: 26884600]

39. ONC201 in Adults With Recurrent H3K27M-mutant Glioma.

40. Oral ONC201 in Recurrent GBM, H3K27M Glioma, and Midline Glioma.

41. ONC201 in Pediatric H3K27M Gliomas.

42. Expanded Access to ONC201 for Patients With H3K27M-mutant and/or Midline High Grade Gliomas.

43. Chi AS, Stafford JM, Sen N, Possemato R, Placantonakis D, Hidalgo ET, et al. H3K27M mutant gliomas are selectively killed by ONC201, a small molecule inhibitor of dopamine receptor D2. Neuro Oncol. 2017;19:vi81. doi:10.1093/neuonc/nox168.334.

44. A phase 2 study of the first imipridone ONC201, a selective DRD2 antagonist for oncology, adminstered every three weeks in recurrent glioblastoma.

45. Chi AS, Tarapore RS, Hall MD, Shonka N, Gardner S, Umemura Y, et al. Pediatric and adult H3K27M-mutant diffuse midline glioma treated with the selective DRD2 antagonist ONC201. J Neurooncol. 2019;145(1):97-105. doi:10.1007/s11060-019-03271-3. [PubMed: 31456142]

46. Hall MD, Odia Y, Allen JE, Tarapore R, Khatib Z, Niazi TN, et al. First clinical experience with DRD2/3 antagonist ONC201 in H3K27M-mutant pediatric diffuse intrinsic pontine glioma: a case report. J Neurosurg Pediatr. 2019:1-7. doi:10.3171/2019.2.PEDS18480.

47. Hornyák L, Dobos N, Koncz G, Karányi Z, Páll D, Szabó Z, et al. The role of indoleamine-2,3dioxygenase in cancer development, diagnostics, and therapy. Front Immunol. 2018;9(151). doi:10.3389/fimmu.2018.00151.

48. Komiya T, Huang CH. Updates in the clinical development of epacadostat and other indoleamine-2,3-dioxygenase 1 inhibitors (IDO1) for human cancers. Front Oncol. 2018;8(423). doi:10.3389/fonc.2018.00423.

49. Lauing KL, Lulla RR, Zhai L, Hashizume R, Fangusaro J, Wainwright DA. IMMU-21. Charactering IDO1 and its therapeutic potential in pediatric central nervous system tumors. Neurooncology. 2017;19(Suppl 4):iv32-iv.

50. Hanihara M, Kawataki T, Oh-Oka K, Mitsuka K, Nakao A, Kinouchi H. Synergistic antitumor effect with indoleamine 2,3-dioxygenase inhibition and temozolomide in a murine glioma model. J Neurosurg. 2016;124(6):1594-601. doi:10.3171/2015.5.JNS141901. [PubMed: 26636389]

51. Johnson TS, Aguilera D, Al-Basheer A, Castellino RC, Eaton BR, Esiashvili N. Front-line therapy of DIPG using the IDO pathway inhibitor indoximod in combination with radiation and chemotherapy. 2018. doi:10.1158/1538-7445.AM2018-CT004.

52. Lieberman NAP, DeGolier K, Kovar HM, Davis A, Hoglund V, Stevens J, et al. Characterization of the immune microenvironment of diffuse intrinsic pontine glioma: implications for development of immunotherapy. Neuro-oncology. 2019;21(1):83-94. doi:10.1093/neuonc/noy145. [PubMed: 30169876] 
53. Lin GL, Nagaraja S, Filbin MG, Suva ML, Vogel H, Monje M. Non-inflammatory tumor microenvironment of diffuse intrinsic pontine glioma. Acta Neuropathol Commun. 2018;6(1):51. doi:10.1186/s40478-018-0553-x. [PubMed: 29954445]

54. Maury E, Hashizume R. Epigenetic modification in chromatin machinery and its deregulation in pediatric brain tumors: Insight into epigenetic therapies. Epigenetics. 2017;12(5):353-369. doi:10.1080/15592294.2016.1278095. [PubMed: 28059591]

55. Majzner RG, Theruvath JL, Nellan A, Heitzeneder S, Cui Y, Mount CW, et al. CAR T cells targeting B7-H3, a pan-cancer antigen, demonstrate potent preclinical activity against pediatric solid tumors and brain tumors. Clin Cancer Res. 2019;25(8):2560-74. doi:10.1158/1078-0432.Ccr-18-0432. [PubMed: 30655315]

56. Tang X, Zhao S, Zhang Y, Wang Y, Zhang Z, Yang M, et al. B7-H3 as a novel CAR-T therapeutic target for glioblastoma. Mol Ther Oncolytics. 2019;14:279-87. doi:10.1016/j.omto.2019.07.002. [PubMed: 31485480]

57. Zhou Z, Singh R, Souweidane MM. Convection-enhanced delivery for diffuse intrinsic pontine glioma treatment. Curr Neuropharmacol. 2017;15(1):116-28. [PubMed: 27306036]

58. Convection-Enhanced Delivery of 124I-8H9 for Patients With Non-Progressive Diffuse Pontine Gliomas Previously Treated With External Beam Radiation Therapy.

59. MTX110 by Convection-Enhanced Delivery in Treating Participants With Newly-Diagnosed Diffuse Intrinsic Pontine Gliome (PNOC015).

60. CED With Irinotecan Liposome Injection Using Real Time Imaging in Children With Diffuse Intrinsic Pontine Glioma (DIPG).

61. Tsvankin V, Hashizume R, Katagi H, Herndon JE, Lascola C, Venkatraman TN, et al. ABC transporter inhibition plus dexamethasone enhances the efficacy of convection enhanced delivery in H3.3K27M mutant diffuse intrinsic pontine glioma. J Neurosurg. 2019. doi:10.1093/neuros/ nyz212.

62. Rees JH. Diagnosis and treatment in neuro-oncology: an oncological perspective. Br J Radiol. 2011;84:S82-S89. doi:10.1259/bjr/18061999. [PubMed: 22433832]

63. Panditharatna E, Kilburn LB, Aboian MS, Kambhampati M, Gordish-Dressman H, Magge SN, et al. Clinically relevant and minimally invasive tumor surveillance of pediatric diffuse midline gliomas using patient-derived liquid biopsy. Clin Cancer Res. 2018;24(23):5850-5859. doi:10.1158/1078-0432.CCR-18-1345. [PubMed: 30322880] • This study illustrates the feasibility of liquid biopsy ctDNA quantification for therapeutic monitoring in DMG patients. ctDNA MAFs in serial CSF and plasma samples were correlated to changes in MRI tumor measurements by ddPCR analysis.

64. Wang Y, Springer S, Zhang M, McMahon KW, Kinde I, Dobbyn L, et al. Detection of tumorderived DNA in cerebrospinal fluid of patients with primary tumors of the brain and spinal cord. Proc Natl Acad Sci USA. 2015;112:9704-9709. doi:10.1073/pnas.1511694112. [PubMed: 26195750]

65. Xu R, Wei W, Krawczyk M, Wang W, Luo H, Flagg K, et al. Circulating tumour DNA methylation markers for diagnosis and prognosis of hepatocellular carcinoma. Nat Mater. 2017;16:1155-1161. doi:10.1038/NMAT4997. [PubMed: 29035356]

66. Pérez-Callejo D, Romero A, Provencio M, Torrente M. Liquid biopsy based biomarkers in nonsmall cell lung cancer for diagnosis and treatment monitoring. Transl Lung Cancer Res. 2016;5(5):455-465. doi:10.21037/tlcr.2016.10.07. [PubMed: 27826527]

67. Dawson SJ, Tsui DWY, Murtaza M, Biggs H, Rueda OM, Chin SF, et al. Analysis of circulating tumor DNA to monitor metastatic breast cancer. New Engl J Med. 2013;368:1199-1209. doi:10.1056/NEJMoa1213261. [PubMed: 23484797]

68. Olmedillas-Lopez S, Garcia-Arranz M, Garcia-Olmo D. Current and emerging applications of droplet digital PCR in oncology. Mol Diagn Ther. 2017;21:493-510. doi:10.1007/ s40291-017-0278-8. [PubMed: 28477149]

69. Pan C, Liu J, Tang J, Chen X, Chen F, Wu Y, et al. A machine learning-based prediction model of H3K27M mutations in brainstem gliomas using conventional MRI and clinical features. Radiother Oncol. 2018;130:172-179. doi:10.1016/j.radonc.2018.07.011. [PubMed: 30097251] 
70. Huang TY, Piunti A, Lulla RR, Qi J, Horbinski CM, Tomita T, et al. Detection of histone H3 mutations in cerebrospinal fluid-derived tumor DNA from children with diffuse midline glioma. Acta Neuropathol Commun. 2017;5:28. doi:10.1186/s40478-017-0436-6. [PubMed: 28416018]

71. Miller AM, Shah RH, Pentsova EI, Pourmaleki M, Briggs S, Distefano N, et al. Tracking tumour evolution in glioma through liquid biopsies of cerebrospinal fluid. Nature. 2019;565:654-670. doi:10/1038/s41586-019-0882-3. [PubMed: 30675060] - This study used sequencing analysis to show that the alterations found in CSF ctDNA closely mirrored those of the tumor genome. The researchers identify serial lumbar punctures as a possible diagnostic and therapeutic monitoring tool.

72. Stallard S, Savelieff MG, Wierzbicki K, Mullan B, Miklja Z, Bruzek A, et al. CSF H3F3A K27M circulating tumor DNA copy number quantifies tumor growth and in vitro treatment response. Acta Neuropathol Commun. 2018;6:80. doi:10.1186/s40478-018-0580-7. [PubMed: 30111355]

73. Tie J, Kinde I, Wang Y, Wong HL, Roebert J, Christie M, et al. Circulating tumor DNA as an early marker of therapeutic response in patients with metastatic colorectal cancer. Ann Oncol. 2015;26(8):1715-1722. doi:10.1093/annonc/mdv177. [PubMed: 25851626]

74. Miklja Z, Pasternak A, Stallard S, Nicolaides T, Kline-Nunnally C, Cole B, et al. Molecular profiling and targeted therapy in pediatric gliomas: review and consensus recommendations. Neuro Oncol. 2019. doi:10.1093/neuonc/noz022. 

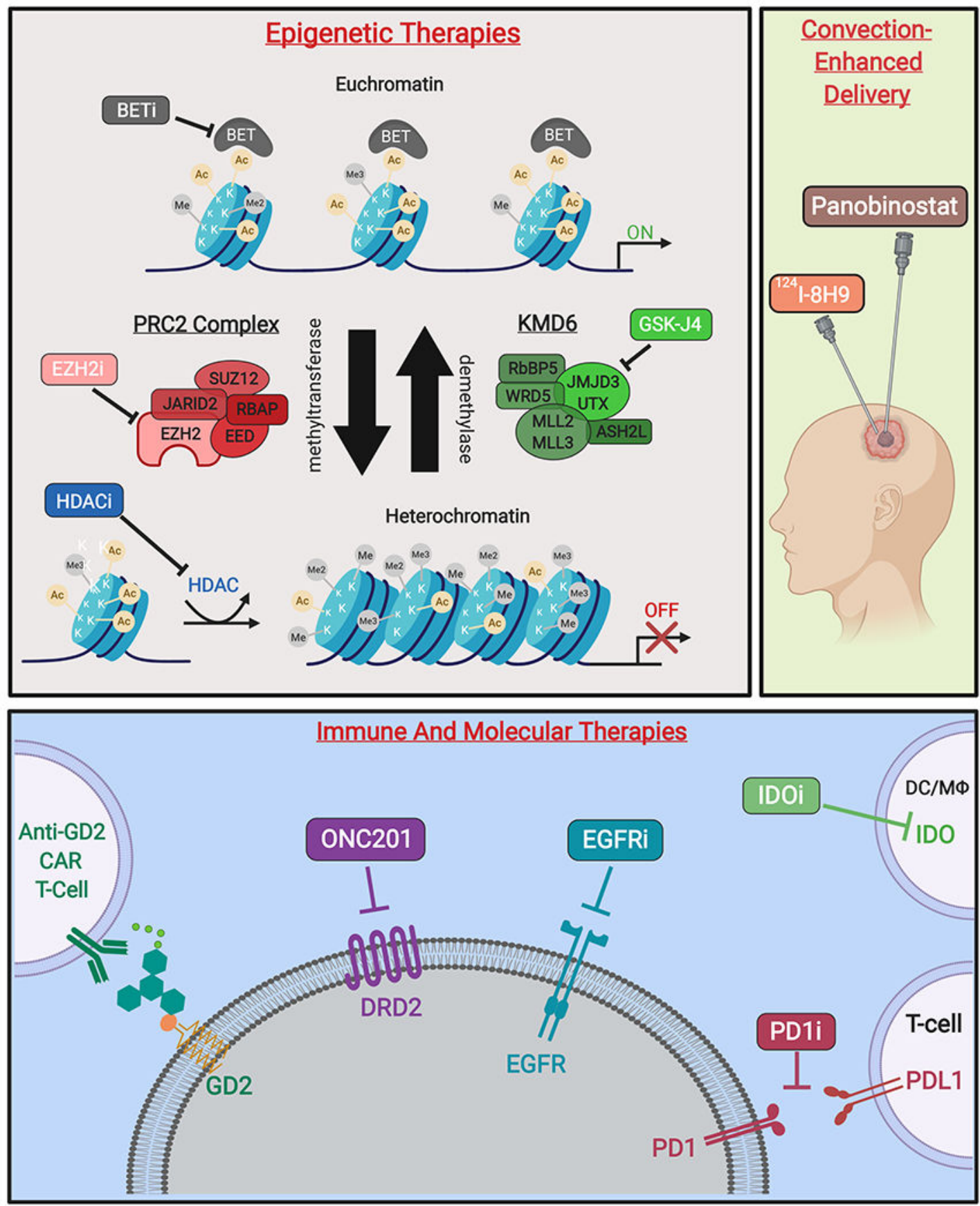

Fig 1.

Novel therapies targeting H3K27M-mutant gliomas. (A)* Epigenetic therapies modify transcriptional activity at the level of chromatin. Competitive inhibition of BET family proteins reduce recognition of acetylated histones and subsequent transcription initiation. Additionally, inhibitors of EZH2 activity prevent formation of heterochromatin by inactivating the catalytic subunit of the PRC2 methyltransferase complex. Moreover, nonspecific HDAC inhibitors reduce heterochromatin formation. Finally, prodrug GSK-J4/J1 increases H3K27 methylation by inhibition of K27-demethylases JMJD3 and UTX. (B) 
Therapeutic agents delivered by CED include omburtamab, a radioactive iodine isotope conjugated to the anti-glioma monoclonal antibody $8 \mathrm{H} 9$ (124I-8H9), which has undergone phase I evaluation [58]. Additionally, delivery of panobinostat nanoparticle formulation MTX110 via CED is ongoing (Phase I/II, PNOC015). (C) Emerging immune therapies include CAR T-cells against GD2, a disialoganglioside highly expressed in H3K27M gliomas. PD1/PDL1 immune checkpoint inhibitors prevent T-cell suppression, while IDO inhibitors increase T-cell activation at the site of tumors cells. Molecular therapies include small-molecule DRD2-antagonist ONC201, as well as inhibitors of EGFR to reduce tumor growth and proliferation [59].

Figure created with BioRender.com. *Figure 1A adapted from Hashizume (2017) [25]. 